\title{
L'ambulatoire avant le stationnaire: première étape du rationnement
}

\author{
Josef E. Brandenberg \\ Dr méd., Président de la fmCh - Organisation faîtière des médecins à activité chirurgicale et invasive
}

Le 13 janvier 2017, le conseiller d'Etat Guido Graf, directeur de la santé du canton de Lucerne, a publié une liste des opérations qui devront obligatoirement être assurées en ambulatoire à partir de l'été 2017.

Cette démarche a été largement saluée dans les médias, mais comme toujours en politique de la santé, au lieu de corriger les erreurs existantes on en commet de nouvelles.

Erreur $n^{\circ}$ 1: Conformément à la loi, les caisses-maladie assument $45 \%$ des coûts des traitements stationnaires et les cantons $55 \%$. Les caisses-maladie paient $100 \%$ des prestations en ambulatoire.

Que les opérations aient lieu en ambulatoire ou dans le domaine stationnaire, les coûts élevés qu'elles impliquent (anesthésie, surveillance, etc.) restent les mêmes. Les caisses-maladie devant payer la totalité du montant pour les interventions en ambulatoire, les primes ne baisseront pas, au contraire, elles vont augmenter. L'automne prochain, on déplorera de nouveau la hausse des coûts dans le secteur ambulatoire, et on en fera porter la responsabilité aux médecins.

La stratégie «l'ambulatoire avant le stationnaire» sert essentiellement à alléger le budget des cantons pour faire face aux restrictions budgétaires actuelles. Elle ne résout pas l'absurdité du financement hospitalier dual fixe.

Erreur $n^{\circ} 2$ : Le principe "Opéré le matin - à la maison le soir même» est présenté comme un avantage pour le patient. La réalité est bien différente. Seule une minorité de patients veulent rentrer chez eux le jour même. Ils préfèrent rester une journée de plus à l'hôpital, parce qu'ils ne se sentent pas encore assez vaillants pour sortir, qu'ils vivent isolés, que les tâches domestiques ne sont pas assurées, ou que personne ne peut les ramener chez eux avant le surlendemain.

Et si l'on regarde à l'étranger, tous les domaines ambulatoires ne se valent pas. Au LDS Hospital, l'hôpital universitaire de Salt Lake City, cela fait déjà des années que l'opération des ligaments croisés se fait en «ambulatoire», dans le Intermountain Medical Center voisin. Après l'intervention, les patients sont hébergés dans l'hôtel affilié, équipé pour les urgences, et ils y passent deux à trois nuits, durant lesquelles ils sont suivis par des infirmières. Aux Pays-Bas, les patients du domaine ambulatoire reçoivent le jour même la visite du personnel soignant des services de soins et d'aide à domicile, ils sont alités et on passe les voir plusieurs fois par jour les jours suivants. Bien sûr, tout cela n'est pas gratuit.

Erreur $n^{\circ} 3$ : Dans le domaine ambulatoire, il n'existe pas (encore) de patients assurés en complémentaire. Jusqu'à maintenant, hôpitaux et médecins s'intéressaient peu aux opérations en ambulatoire. La liste «l'ambulatoire avant le stationnaire" génère des pertes sévères, tout comme le choc du franc fort pour les exportations. Ce n'est pas le secteur de la santé qui va s'en indigner. Les médecins agréés et cliniques privées supportent de toute façon le risque seuls, à titre d'entreprises indépendantes, et ravalent leur colère. Les hôpitaux publics reportent les dépenses sur la communauté. Soit les déficits augmentent, soit les gains devant être assurés au profit du budget public baissent (comme l'an dernier à l'hôpital cantonal de Lucerne LUKS).

Erreur $n^{\circ}$ 4: Il relève du domaine médical de décider si un patient doit ou non être hospitalisé. Ce sont les médecins qui assument la responsabilité de cette décision. Soudain, avec la liste du conseiller d'Etat, l'exigence d'une prise en charge globale, tenant compte du contexte social (patients habitant à la campagne, personnes âgées, etc.), n'a plus d'importance.

Une fois cette liste mise en place, chaque séjour hospitalier nécessaire au niveau médical ou pour les soins devra faire l'objet d'une demande écrite. La bureaucratie va encore augmenter, au détriment des médecins et des hôpitaux.

Erreur $n^{\circ}$ 5: Le conseiller d'Etat Guido Graf a dressé cette liste sans consulter le corps médical. C'est normal, puisqu'il s'agit d'un rationnement. On prive les patients de prestations, pour des raisons financières. Le rationnement ne relève pas de la compétence des médecins. Le rationnement est une décision politique. Avec cette étape du rationnement, le conseiller d'Etat Guido Graf a levé un tabou. 